\title{
Zeros of Random Functions Generated with de Branges Kernels
}

\section{Jorge Antezana ${ }^{1}$, Jordi Marzo ${ }^{2, *}$ and Jan-Fredrik Olsen ${ }^{3}$}

${ }^{1}$ Instituto Argentino de Matemática "Alberto P. Calderón," and

Universidad Nacional de La Plata, Departamento de Matemática, Esq. 50 y 115 s/n, Facultad de Ciencias Exactas de La Plata (1900), Buenos Aires, Argentina, ${ }^{2}$ Departament de matemàtiques i informàtica, Universitat de Barcelona and BGSMath, Gran Via 585, 08007, Barcelona, Spain, and ${ }^{3}$ Centre for Mathematical Sciences, Lund University, P.O. Box 118, SE-221 00 Lund, Sweden

*Correspondence to be sent to: e-mail: jmarzo@ub.edu

We study the point process given by the set of real zeros of random series generated with orthonormal bases of reproducing kernels of de Branges spaces. We find an explicit formula for the intensity function in terms of the phase of the Hermite-Biehler function generating the de Branges space. We prove that the intensity of the point process completely characterizes the underlying de Branges space.

\section{Introduction}

\section{1 de Branges spaces}

Let $E(z)$ be a function of the Hermite-Biehler class, that is, $E(z)$ is entire and satisfies the inequality $|E(z)|>\left|E^{*}(z)\right|$ for $\operatorname{Im} z>0$, where $E^{*}(z)=\overline{E(\bar{z})}$. Given such a function, the de Branges space $H(E)$ is defined by

$$
H(E)=\left\{f \text { entire }: \frac{f}{E^{\prime}}, \frac{f}{E^{*}} \in H^{2}\left(\mathbb{C}_{+}\right)\right\}
$$

Received April 30, 2015; Revised March 24, 2016; Accepted April 8, 2016

Communicated by Misha Sodin

(C) The Author(s) 2016. Published by Oxford University Press. All rights reserved. For permissions, please e-mail: journals.permission@oup.com. 
2 J. Antezana et al.

with norm given by

$$
\|f\|_{H(E)}^{2}=\int_{-\infty}^{+\infty}\left|\frac{f(x)}{E(x)}\right|^{2} \mathrm{~d} x
$$

Here, we use $H^{2}\left(\mathbb{C}_{+}\right)$to denote the Hardy space of the upper half-plane.

The de Branges spaces generalize the classical Paley-Wiener space, obtained by letting $E(z)=\mathrm{e}^{-\mathrm{i} z}$, and are used in the study of differential equations. The standard reference for de Branges spaces is the book [4] by de Branges.

We consider Hermite-Biehler functions without real zeros. This excludes the existence of points $x \in \mathbb{R}$ such that $f(x)=0$ for all $f \in H(E)$.

Recall that a function $K_{W}$ is the reproducing kernel for a space $H(E)$ for the point $w \in \mathbb{C}$, if, for all $f \in H(E)$,

$$
f(W)=\left\langle f, K_{W}\right\rangle_{H(E)}=\int_{-\infty}^{+\infty} f(x) \overline{K_{W}(x)} \frac{\mathrm{d} x}{|E(x)|^{2}} .
$$

This function exists since point evaluations are bounded functionals. Moreover,

$$
K_{W}(z)=K(z, w)=\frac{\mathrm{i}}{2 \pi} \frac{E(z) \overline{E(W)}-E^{*}(z) \overline{E^{*}(W)}}{z-\bar{W}}, \quad z \neq w .
$$

As it is usual, we let $k_{W}=K_{W} /\left\|K_{W}\right\|_{H(E)}$ denote the normalized reproducing kernels.

Since the metric structure of $H(E)$ is defined on the real line, for $x \in \mathbb{R}$, it is useful to introduce the polar decomposition $E(x)=|E(x)| \mathrm{e}^{-\mathrm{i} \phi(x)}$. The so-called phase function $\phi$ is a strictly increasing $C^{\infty}(\mathbb{R})$ function (see [4, Problem 48]). With this notation, we have

$$
\left\|K_{X}\right\|^{2}=K(x, x)=\frac{1}{\pi} \phi^{\prime}(x)|E(x)|^{2},
$$

whence, for $x, y \in \mathbb{R}$, with $x \neq y$,

$$
k_{Y}(x)=\frac{K(x, y)}{K(y, y)^{1 / 2}}=\frac{|E(x)|}{\sqrt{\pi \phi^{\prime}(y)}} \frac{\sin (\phi(x)-\phi(y))}{x-y} .
$$

(In the case of the Paley-Wiener space, this is exactly a translate of the cardinal sine function.)

Observe that $k_{Y}(x)=\left\langle k_{y}, K_{X}\right\rangle=0$ whenever $x, y \in \mathbb{R}$ are such that $\phi(x)-\phi(y)=k \pi$ for $k \in \mathbb{Z}$. This means that if $\left\{\omega_{n}\right\}$ is the sequence of points such that $\phi\left(\omega_{n}\right)=\alpha+\pi n$, for some $\alpha \in[0, \pi)$, then the family of functions $\left\{k_{\omega_{n}}\right\}$ forms an orthonormal system in $H(E)$. In fact, by the following result of de Branges ([4, p. 55]), a sequence of points 
$\left\{\omega_{n}\right\}$ almost always yields an orthogonal basis of reproducing kernels if and only if $\phi\left(\omega_{n+1}\right)-\phi\left(\omega_{n}\right)=\pi n$ for all $n$.

Theorem 1.1. Suppose that $H(E)$ is a de Branges space and that $\omega_{n}$ are the points defined above. Then, for all $\alpha \in[0, \pi)$, except at most one, the system $\left\{k_{\omega_{n}}\right\}$ is an orthonormal basis for $H(E)$. Moreover, these are the only orthonormal bases of reproducing kernels, and the exceptional $\alpha$ is characterized by the condition that $\mathrm{e}^{\mathrm{i} \alpha z} E(z)-\mathrm{e}^{-\mathrm{i} \alpha z} E^{*}(z) \in$ $H(E)$.

\section{2 de Branges GAFs}

Let $\left\{\psi_{n}\right\}$ be a sequence of functions defined and analytic in a region $\Lambda \subset \mathbb{C}$ symmetric with respect to the real line. If the $\psi_{n}$ are real valued on $\mathbb{R}$ and symmetric on $\Lambda$ (i.e., $\left.\overline{\psi_{n}(z)}=\psi_{n}(\bar{z})\right), \sum_{n}\left|\psi_{n}(z)\right|^{2}$ converges uniformly on compact subsets of $\Lambda$, and $a_{n}$ are real i.i.d. standard normal random variables, then the function

$$
\Psi(z)=\sum_{n} a_{n} \psi_{n}(z)
$$

is called a symmetric Gaussian Analytic Function (symmetric GAF) (see [6, Definition 1.1]).

A symmetric GAF is a holomorphic function with probability one (see [7, Lemma 2.2.3] for a proof in the closely related case of complex GAFs).

In this article, we consider random functions constructed using orthonormal bases of reproducing kernels.

Definition 1.2. Let $H(E)$ be a de Branges space with an orthonormal basis of reproducing kernels $\left\{k_{\omega_{n}}\right\}$. For real i.i.d. standard normal random variables $a_{n}$, a de Branges GAF is the random function

$$
F(x)=\sum_{n} a_{n} k_{\omega_{n}}(x)
$$

It is easy to see that a de Branges GAF is a symmetric GAF.

The main feature connecting the de Branges GAF $F(x)$ with the de Branges space $H(E)$ is that, by definition, the covariance kernel of the gaussian process $(F(x))_{x \in \mathbb{R}}$ 
coincides with the reproducing kernel of the de Branges space $H(E)$, that is,

$$
\mathbb{E}[F(x) F(y)]=K(x, y),
$$

where $\mathbb{E}$ denotes the expectation. Recall that the covariance kernel, as $(F(x))_{x \in \mathbb{R}}$ has mean zero, completely defines the behavior of the process. In particular, this means that the choice of basis is irrelevant.

\subsection{The intensity function $\rho$}

Let $Z_{\mathbb{R}}(\Psi)$ denote the set of real zeros of a symmetric GAF defined as in (3). We define the intensity function of the process by the relation

$$
\mathbb{E}\left[\#\left(Z_{\mathbb{R}}(\Psi) \cap I\right)\right]=\int_{I} \rho(x) \mathrm{d} x,
$$

where $I$ is any open interval in $\mathbb{R}$.

We remark that we should perhaps call the function $\rho(x)$ defined on $\mathbb{R}$ in this way the real intensity function of the symmetric GAF to separate it from the more usual definition, where the intervals $I \subset \mathbb{R}$ are replaced by open sets $D \subset \mathbb{C}$, and $Z_{\mathbb{R}}(\Psi)$ is replaced by the set of complex zeros $Z_{\mathbb{C}}(\Psi)$. By our logic, we should call the resulting intensities defined on $\mathbb{C}$ complex intensity functions. We denote them by $\rho_{\mathbb{C}}(z)$.

In the setting of symmetric GAFs, Feldheim [6] proved that in the sense of distributions

$$
\rho_{\mathbb{C}}(z)=\frac{1}{\pi} \Delta \log \left(K(z, z)+\sqrt{K(z, z)^{2}-|K(z, \bar{z})|^{2}}\right) .
$$

As in the case of zeros of random polynomials with real coefficients, it is to be expected that symmetric GAFs have a positive proportion of their zeros on the real line. Indeed, it follows from (5) that the complex intensity function gives rise to a singular part on the real line that is absolutely continuous with respect to one-dimensional Lebesgue measure. When computing this density, one obtains the Edelman-Kostlan formula

$$
\rho(x)=\frac{1}{\pi} \sqrt{\left.\frac{\partial^{2}}{\partial t \partial s} \log K(t, s)\right|_{t=s=x}},
$$

(cf. [5, Theorem 3.1] and [9]).

In Theorem 2.1, we give a formula for the intensity function for de Branges GAFs using the formula (6) as a starting point. Our main results, Theorems 2.2 and 2.3 , are analogous to rigidity results due to Sodin in the setting of complex GAFs. They 
say that if the point processes associated to the zeros of two de Branges GAFs have the same intensity functions, then the corresponding de Branges GAFs have the same distribution. In particular, this means that the distribution of the real zeros determine the distribution of the complex zeros. This is not true for symmetric GAFs in general as there are several different stationary processes, which all have constant intensity.

\subsection{Structure of the article and notation}

The main results are in Section 2, and their proofs are in Sections 3 and 4. Throughout this article we use the notation $f(x) \lesssim g(x)$ to indicate that $f(x) / g(x)$ is bounded above by some positive constant. We write $f(x) \simeq g(x)$ if both $f(x) \lesssim g(x)$ and $g(x) \lesssim f(x)$ hold.

\section{The Main Results}

\subsection{A formula for the intensity function}

In the following theorem, we give a formula for the intensity function of de Branges GAFs in terms of the Schwarzian derivative of the phase function $\phi$, that is,

$$
S[\phi]=\left(\frac{\phi^{\prime \prime}}{\phi^{\prime}}\right)^{\prime}-\frac{1}{2}\left(\frac{\phi^{\prime \prime}}{\phi^{\prime}}\right)^{2} .
$$

Theorem 2.1. Let $H(E)$ be a de Branges space with phase function $\phi$. Then the intensity function of the corresponding de Branges GAF satisfies

$$
\rho(x)=\frac{1}{\pi} \sqrt{\frac{\phi^{\prime}(x)^{2}}{3}+\frac{S[\phi](x)}{6}} .
$$

The Schwarzian derivative can be seen as an infinitesimal version of the crossratio (see [2]). It is invariant under Moebius transformations and it measures, in some sense, how far a map is from being a Moebius map. Geometrically, it is connected with curvature, see the survey paper [8].

\subsection{A rigidity result for de Branges GAFs}

An interesting feature in the related setting of complex GAFs is that the distribution of the zero set depends only on the complex intensity function, as was shown by Sodin in [9] (see also [7, Section 2.5.]). 
In our case, as we are working in a (symmetric) real context, Sodin result does not apply. However, as we show in the following theorem, the intensity of the real zeros, although does not allow to recover the underlying space, still determines the distribution of zeros of the de Branges GAFs.

Theorem 2.2. Let $F, G$ be two de Branges GAFs and let $\rho^{F}(x), \rho^{G}(x)$ be the respective intensity functions. If, for all $x \in \mathbb{R}$,

$$
\rho^{F}(x)=\rho^{G}(x)
$$

then there exists a non-random analytic function $S$, which does not vanish anywhere, such that $S F$ and $G$ have the same distribution. In particular, the random point processes given by the zeros of $F$ and $G$ have the same distribution.

This result follows as a consequence of the following theorem, which shows that the intensity function not only determines the de Branges GAF but also the underlying de Branges space up to a very special isometric isomorphism.

Theorem 2.3. Let $E_{1}, E_{2}$ be Hermite-Biehler functions, $K_{1}(z, w)$ and $K_{2}(z, w)$ be the corresponding reproducing kernels of the de Branges spaces $H\left(E_{1}\right), H\left(E_{2}\right)$, and $\rho^{E_{1}}, \rho^{E_{2}}$ be the respective intensity functions. If for all $x \in \mathbb{R}$

$$
\rho^{E_{1}}(x)=\rho^{E_{2}}(x),
$$

then there exists an entire function $S$ without zeros, such that

$$
K_{2}(z, w)=S(z) K_{1}(z, w) \overline{S(W)},
$$

and the $\operatorname{map} f \mapsto S f$ is an isometry from $H\left(E_{1}\right)$ to $H\left(E_{2}\right)$,

Observe that it follows from this result that a de Branges GAF is not stationary unless the kernel is essentially the reproducing kernel of a Paley-Wiener space (see [7, Corollary 2.5.4.]). 


\section{Proof of Theorem 2.1}

As we mentioned Section 1, the first intensity of the real zeros can be computed using the Edelman-Kostlan formula [5, Theorem 3.1]:

$$
\rho(x)=\frac{1}{\pi} \sqrt{\left.\frac{\partial^{2}}{\partial t \partial s} \log K(s, t)\right|_{s=t=x}} .
$$

As the covariance kernel for the Gaussian process is the reproducing kernel for a space of analytic functions, following Bergman [3, p. 35, formula (27)], this quantity is related with an extremal problem

$$
\left.\frac{\partial^{2}}{\partial t \partial s} \log K(t, s)\right|_{s=t=x}=K(x, x) \frac{\inf \left\{\|h\|_{H(E)}^{2}: h(x)=1\right\}^{2}}{\inf \left\{\|h\|_{H(E)}^{2}: h(x)=0, h^{\prime}(x)=1\right\}}
$$

where it is implicit that both infimums are taken only over $h \in H(E)$. This can be reformulated as

$$
\left.\frac{\partial^{2}}{\partial t \partial s} \log K(t, s)\right|_{s=t=x}=\frac{\sup \left\{\left|h^{\prime}(x)\right|^{2}:\|h\|_{H(E)}=0, h(x)=1\right\}}{K(x, X)} .
$$

Using (8), we can compute $\rho(x)$ by solving this (deterministic) extremal problem in $H(E)$. To this end, we prove the following technical lemma.

Lemma 3.1. Let $H(E)$ be a de Branges space with phase function $\phi$. If $\left\{k_{\omega_{n}}\right\}$ is an orthonormal basis of reproducing kernels for $H(E)$, then

$$
\sum_{n \neq k} \frac{\phi^{\prime}\left(\omega_{k}\right)}{\left(\omega_{k}-\omega_{n}\right)^{2} \phi^{\prime}\left(\omega_{n}\right)}=\frac{\phi^{\prime}\left(\omega_{k}\right)^{2}}{3}+\frac{S[\phi]\left(\omega_{k}\right)}{6}
$$

Proof. Fix $n$, and let $x \in \mathbb{R} \backslash\left\{\omega_{n}\right\}$. Combining the well-known formula

$$
\sum\left|k_{\omega_{n}}(x)\right|^{2}=K(x, x)
$$

with (1) and (2), we obtain

$$
\sum_{n} \frac{1}{\phi^{\prime}\left(\omega_{n}\right)\left(x-\omega_{n}\right)^{2}}=\frac{\phi^{\prime}(x)}{\sin ^{2}(\alpha-\phi(x))}
$$


whence

$$
\begin{aligned}
\sum_{n \neq k} \frac{\phi^{\prime}\left(\omega_{k}\right)}{\phi^{\prime}\left(\omega_{n}\right)\left(x-\omega_{n}\right)^{2}}= & \frac{\phi^{\prime}\left(\omega_{k}\right) \phi^{\prime}(x)}{\sin ^{2}\left(\phi\left(\omega_{k}\right)-\phi(x)\right)}-\frac{1}{\left(x-\omega_{k}\right)^{2}} \\
= & \left(\frac{\phi^{\prime}\left(\omega_{k}\right) \phi^{\prime}(x)}{\sin ^{2}\left(\phi\left(\omega_{k}\right)-\phi(x)\right)}-\frac{\phi^{\prime}\left(\omega_{k}\right) \phi^{\prime}(x)}{\left(\phi\left(\omega_{k}\right)-\phi(x)\right)^{2}}\right) \\
& +\left(\frac{\phi^{\prime}\left(\omega_{k}\right) \phi^{\prime}(x)}{\left(\phi\left(\omega_{k}\right)-\phi(x)\right)^{2}}-\frac{1}{\left(x-\omega_{k}\right)^{2}}\right) .
\end{aligned}
$$

The first summand converges to $\phi^{\prime}\left(\omega_{k}\right)^{2} / 3$ as $x \rightarrow \omega_{k}$ since

$$
\lim _{x \rightarrow 0}\left(\frac{1}{\sin ^{2}(x)}-\frac{1}{x^{2}}\right)=\frac{1}{3} .
$$

To deal with the second summand, we use the known formula

$$
\lim _{x, y \rightarrow t} \frac{\phi^{\prime}(x) \phi^{\prime}(x)}{(\phi(x)-\phi(y))^{2}}-\frac{1}{(x-y)^{2}}=\frac{1}{6} S[\phi](t) .
$$

It follows from Theorem 1.1 that for almost every $x \in \mathbb{R}$, there exists a sequence $\left\{\omega_{n}\right\} \subset \mathbb{R}$ such that $\left\{k_{\omega_{n}}\right\}$ is an orthonormal basis for $H(E)$ and $x=\omega_{k}$ for some $k \in \mathbb{Z}$. Hence, for such an $x$, we fix the corresponding sequence $\left\{\omega_{n}\right\}$ and use the notation $x=\omega_{k}$.

Our first step is to rewrite the variational formulation of the Bergman metric in the following way:

$$
\begin{aligned}
\rho\left(\omega_{k}\right)^{2} & =\frac{\sup \left\{\left|h^{\prime}\left(\omega_{k}\right)\right|^{2}:\|h\|_{H(E)}=1, h\left(\omega_{k}\right)=0\right\}}{\pi^{2} K\left(\omega_{k}, \omega_{k}\right)} \\
& =\frac{1}{\pi \phi^{\prime}\left(\omega_{k}\right)} \sup \left\{\left|\frac{h^{\prime}\left(\omega_{k}\right)}{E\left(\omega_{k}\right)}\right|^{2}:\|h\|_{H(E)}=1, h\left(\omega_{k}\right)=0\right\} \\
& =\frac{1}{\pi \phi^{\prime}\left(\omega_{k}\right)} \sup \left\{\left|\left(\frac{h}{E}\right)^{\prime}\left(\omega_{k}\right)\right|^{2}:\|h\|_{H(E)}=1, h\left(\omega_{k}\right)=0\right\} .
\end{aligned}
$$

Let $h \in H(E)$ satisfy $\|h\|_{H(E)}=1$ and $h\left(\omega_{k}\right)=0$. Since the functions

$$
k_{\omega_{n}}(u)=\frac{|E(x)| \sin \left(\phi(u)-\phi\left(\omega_{n}\right)\right)}{\sqrt{\pi \phi^{\prime}\left(\omega_{n}\right)}\left(u-\omega_{n}\right)},
$$

form an orthonormal basis for $H(E)$, there exists a sequence $\left\{c_{n}\right\} \in \ell^{2}(\mathbb{Z})$ such that

$$
\begin{aligned}
\frac{h(u)}{E(u)}=\sum_{n \neq k} c_{n} \frac{k_{\omega_{n}}(u)}{E(u)} & =\sum_{n \neq k} c_{n} \frac{\sin \left(\phi(u)-\phi\left(\omega_{n}\right)\right)}{\sqrt{\pi \phi^{\prime}\left(\omega_{n}\right)}\left(u-\omega_{n}\right) \mathrm{e}^{-\mathrm{i} \phi(u)}} \\
& =\frac{1}{2 \sqrt{\pi}} \sum_{n \neq k} c_{n}\left(i \mathrm{e}^{\mathrm{i} \alpha}(-1)^{n}\right) \frac{\left(1-\mathrm{e}^{2 i(\phi(u)-\alpha)}\right)}{\sqrt{\phi^{\prime}\left(\omega_{n}\right)}\left(u-\omega_{n}\right)} .
\end{aligned}
$$


Here, we used that $\phi\left(\omega_{k}\right)=\alpha+\pi k$. As we may assume that the sequence $c_{n}$ is finite, we can differentiate term-by-term to get

$$
\left(\frac{h}{E}\right)^{\prime}(u)=\frac{1}{\sqrt{\pi}} \sum_{n \neq k} \tilde{c}_{n}\left(\frac{\phi^{\prime}(u) \mathrm{e}^{2 \mathrm{i}(\phi(u)-\alpha)}}{\sqrt{\phi^{\prime}\left(\omega_{n}\right)}\left(u-\omega_{n}\right)}\right)-\sum_{n \neq k} c_{n}\left(\frac{k_{\omega_{n}}(u)}{E(u)\left(u-\omega_{n}\right)}\right)
$$

where $\tilde{c}_{n}=c_{n}\left(\mathrm{e}^{\mathrm{i} \alpha}(-1)^{n}\right)$. We now choose $u=\omega_{k}$. Since $k_{\omega_{n}}\left(\omega_{k}\right)=0$ for every $n \neq k$, it holds that

$$
\left(\frac{h}{E}\right)^{\prime}\left(\omega_{k}\right)=\frac{1}{\sqrt{\pi}} \sum_{n \neq k} \tilde{C}_{n}\left(\frac{\phi^{\prime}\left(\omega_{k}\right)}{\sqrt{\phi^{\prime}\left(\omega_{n}\right)}\left(\omega_{k}-\omega_{n}\right)}\right)
$$

Therefore, the supremum in (10) can be rewritten as

$$
\rho\left(\omega_{k}\right)^{2}=\frac{\phi^{\prime}\left(\omega_{k}\right)}{\pi^{2}} \sup \left\{\left|\sum_{n \neq k} \frac{d_{n}}{\sqrt{\phi^{\prime}\left(\omega_{n}\right)}\left(\omega_{k}-\omega_{n}\right)}\right|^{2}: d_{k}=0, \quad \sum_{n \in \mathbb{Z}}\left|d_{n}\right|^{2}=1\right\} .
$$

As this is the dual formulation of an $\ell^{2}$-norm, it follows immediately that

$$
\rho\left(\omega_{k}\right)^{2}=\frac{\phi^{\prime}\left(\omega_{k}\right)}{\pi^{2}} \sum_{n \neq k} \frac{1}{\phi^{\prime}\left(\omega_{n}\right)\left(\omega_{k}-\omega_{n}\right)^{2}}
$$

Since $x=\omega_{k}$, by Lemma 3.1, we get Theorem 2.1.

Remark 3.2. Formula (7) for $\rho$ can be obtained also by using (5) and computing its singular part (6) or by using, the so called, Rice formula (see [1, Chapter 11]):

$$
\rho(x)=\mathbb{E}\left[\left|F_{\Theta}^{\prime}(x)\right|: F_{\Theta}(x)=0\right]=\int_{\mathbb{R}}|t| p_{F_{\Theta}(x), F_{\Theta}^{\prime}(x)}(0, t) \mathrm{d} t,
$$


$10 \mathrm{~J}$. Antezana et al.

where $F_{\Theta}(x)=\frac{F(x)}{E(x)}$, and the function $p_{F_{\Theta}(x), F_{\Theta}^{\prime}(x)}$ is the joint probability density of the two-dimensional normal vector $\left(F_{\Theta}(x), F_{\Theta}^{\prime}(x)\right)$ with covariance matrix

$$
\left(\begin{array}{cc}
\mathbb{E}\left[F_{\Theta}(x)^{2}\right] & \mathbb{E}\left[F_{\Theta}(x) F_{\Theta}^{\prime}(x)\right] \\
\mathbb{E}\left[F_{\Theta}(x) F_{\Theta}^{\prime}(x)\right] & \mathbb{E}\left[F_{\Theta}^{\prime}(x)^{2}\right]
\end{array}\right) .
$$

\section{Proof of Theorems 2.2 and 2.3}

We begin with a lemma on the Schwarzian derivative. It is one of four lemmas from which Theorem 2.3 follows.

Lemma 4.1. Suppose that $\phi_{1}, \phi_{2} \in \mathbb{C}^{\infty}(\mathbb{R})$ are strictly monotone and that for all $x \in \mathbb{R}$,

$$
S\left[\phi_{1}\right](x)+2 \phi_{1}^{\prime}(x)^{2}=S\left[\phi_{2}\right](x)+2 \phi_{2}^{\prime}(x)^{2} .
$$

If we put $\psi=\phi_{2} \circ \phi_{1}^{-1}$, then it holds that

$$
1=\psi^{\prime}(t)^{2}+\frac{1}{2} S[\psi](t)
$$

Proof. The Chain Rule for the Schwarzian derivative is

$$
S[f \circ g]=(S[f] \circ g)\left(g^{\prime}\right)^{2}+S[g],
$$

whence

$$
S\left[\phi_{1}\right]\left(\phi_{1}^{-1}(t)\right)=-\frac{S\left[\phi_{1}^{-1}\right](t)}{\left(\phi_{1}^{-1}\right)^{\prime}(t)^{2}} .
$$

Combined with the usual chain rule, this yields

$$
2 \phi_{1}^{\prime}\left(\phi_{1}^{-1}(t)\right)^{2}+S\left[\phi_{1}\right]\left(\phi_{1}^{-1}(t)\right)=\frac{2}{\left(\phi_{1}^{-1}\right)^{\prime}(t)^{2}}-\frac{S\left[\phi_{1}^{-1}\right](t)}{\left(\phi_{1}^{-1}\right)^{\prime}(t)^{2}}
$$

Applying the hypothesis to the left-hand side above, with $x=\phi_{1}^{-1}(t)$, and rearranging the terms, this becomes

$$
2=2 \phi_{2}^{\prime}\left(\phi_{1}^{-1}(t)\right)^{2}\left(\phi_{1}^{-1}\right)^{\prime}(t)^{2}+S\left[\phi_{2}\right]\left(\phi_{1}^{-1}(t)\right)\left(\phi_{1}^{-1}\right)^{\prime}(t)^{2}+S\left[\phi_{1}^{-1}\right](t) .
$$


Now, using both chain rules on the right-hand side, we get

$$
2=2\left(\phi_{2} \circ \phi_{1}^{-1}\right)^{\prime}(t)^{2}+S\left[\phi_{2} \circ \phi_{1}^{-1}\right](t)
$$

whence the result follows.

Let $\psi$ be as in the previous lemma. If we define

$$
u(t)=\psi^{\prime}(t) \quad \text { and } \quad v(t)=\frac{1}{2} \frac{\psi^{\prime \prime}(t)}{\psi^{\prime}(t)}
$$

then these new functions satisfy the autonomous system

$$
\left\{\begin{array}{l}
u^{\prime}=2 u v \\
v^{\prime}=1-u^{2}+v^{2} .
\end{array}\right.
$$

Hence, in order to study the solutions of the differential equation (13), we first study some properties of the solutions of this system. To this end, we consider a two smooth functions $u(t)$ and $v(t)$, defined in some common domain, that satisfy (14) and the initial condition

$$
\left(u\left(t_{0}\right), v\left(t_{0}\right)\right)=\left(x_{0}, y_{0}\right) \quad \text { with } x_{0}>0 .
$$

Multiplying both sides of the second equation of (14) by $2 u v$ and then applying the first equation of (14), we obtain

$$
2 u v v^{\prime}=u^{\prime}-u^{2} u^{\prime}+u^{\prime} v^{2}
$$

So, by adding $2 u^{2} u^{\prime}$ to both sides of the equality we get

$$
\left(2 v v^{\prime}+2 u u^{\prime}\right) u=u^{\prime}\left(u^{2}+v^{2}+1\right)
$$

If we define, on the right half-plane $\left\{(x, y) \in \mathbb{R}^{2}: x>0\right\}$, the function

$$
H(x, y)=\frac{\left(x^{2}+y^{2}+1\right)}{x},
$$

the identity (15) shows that

$$
\left.\frac{\mathrm{d}}{\mathrm{d} t} H(u(t), v(t))\right)=0
$$


12 J. Antezana et al.

Therefore, for every $t$ the vector $(u(t), v(t))$ belongs to the same level set of the function $H$. A simple computation shows that $H(x, y) \geq 1$ and for every $c \geq 1$, the level set $H(x, y)=c$ is the circle

$$
(x-c)^{2}+y^{2}=c^{2}-1 .
$$

Now, as the system is regular, classical results on existence and uniqueness of solutions for systems of ODEs, and the compactness of the circles imply that any solution of (14) with initial data in the right half plane can be extended to a periodic solution that stays within the same level set of $H$ (and in particular, stays in the right half-plane).

From now on, when we consider a solutions of (14), we will assume that it is already extended by periodicity to the whole real line. For our problem, we need to know more information about the period of the solutions.

Lemma 4.2. Suppose that $(u(t), v(t))$ is a solution of the system (14) with initial data in the right half plane. Then

$$
u(t+\pi)=u(t) \quad \text { and } \quad v(t+\pi)=v(t) .
$$

Proof. If $\left(u\left(t_{0}\right), v\left(t_{0}\right)\right)=(1,0)$, then (14) implies that the functions $u, v$ are constant whence the lemma is trivially true. We may therefore assume that the solution $(u(t), v(t))$ belongs to the circle

$$
(x-c)^{2}+y^{2}=c^{2}-1,
$$

for some $c>1$ and every $t \in \mathbb{R}$. We prove the result, under this assumption, for $u(t)$.

Let $T$ denote the period of $u$, and let

$$
x_{-}=c-\sqrt{c^{2}-1} \text { and } x_{+}=c+\sqrt{c^{2}-1}
$$

be the points where the circle (16) intersects the $x$-axis. If $u(\tilde{t})=x_{-}$, then, by the symmetries of (14), from time $\tilde{t}$ to time $\tilde{t}+T / 2$ the function $u(t)$ goes from $x_{-}$to $x_{+}$. Moreover, we can also assume that it moves through the upper part of the circle. That is, except for $t=\tilde{t}$ and $t=\tilde{t}+T / 2$, we have

$$
u^{\prime}(t)=2 u(t) \sqrt{\left(c^{2}-1\right)-(u(t)-c)^{2}}>0 .
$$


So, by the inverse change of variable $x=u(t)$, we get that

$$
\begin{aligned}
\frac{T}{2}=\int_{\tilde{t}}^{\tilde{t}+T / 2} \mathrm{~d} t & =\int_{x_{-}}^{x_{+}} \frac{\mathrm{d} x}{2 x \sqrt{\left(c^{2}-1\right)-(x-c)^{2}}} \\
& =\int_{1 / x_{-}}^{1 / x_{+}} \frac{-\mathrm{d} z}{2 \sqrt{\left(c^{2}-1\right)-(z-c)^{2}}} \\
& =-\frac{1}{2}\left[\arcsin \left(\frac{z-c}{\sqrt{c^{2}-1}}\right)\right]_{1 / x_{-}}^{1 / x_{+}}=\frac{\pi}{2},
\end{aligned}
$$

where, in the second identity, we used the change of variables $z=1 / x$.

Lemma 4.3. Suppose that $(u(t), v(t))$ is a solution of the system (14) such that $v(0)=0$ and $u(t)>0$ for some (every) $t>0$, and let

$$
U(s)=\int_{0}^{s} u(t) \mathrm{d} t
$$

Then, for all $k \in \mathbb{Z}$, we have

$$
U\left(k \frac{\pi}{2}\right)=k \frac{\pi}{2} .
$$

Proof. If $u(0)=1$, then $u(t)=1$ for every $t$ and the assertion is trivially true. So, as in the previous lemma, we assume that the solution $(u(t), v(t))$ belongs to the circle

$$
(x-c)^{2}+y^{2}=c^{2}-1,
$$

for some $c>1$. Using, the change of variable $x=u(t)$ as above, followed by the change of variables $s=(x-c) / \sqrt{c^{2}-1}$, we get

$$
U\left(\frac{\pi}{2}\right)=\frac{1}{2} \int_{x_{-}}^{x_{+}} \frac{\mathrm{d} x}{\sqrt{\left(c^{2}-1\right)-(x-c)^{2}}}=\frac{1}{2} \int_{-1}^{1} \frac{\mathrm{d} s}{\sqrt{1-s^{2}}}=\frac{\pi}{2} .
$$

Proof of Theorem 2.3. Let $\phi_{1}(x)$ and $\phi_{2}(x)$ be the phase functions corresponding to the Hermite-Biehler functions $E_{1}(z), E_{2}(z)$. As $\rho^{E_{1}}(x)=\rho^{E_{2}}(x)$, we get by Lemma 4.1 that the function $\left(\phi_{2} \circ \phi_{1}^{-1}\right)(t)$ solves equation (13).

Let $\alpha \in \mathbb{R}$ be such that $\left(\phi_{2} \circ \phi_{1}^{-1}\right)^{\prime \prime}(\alpha)=0$. Indeed, such an $\alpha$ exists because $\left(\psi^{\prime}, \psi^{\prime \prime} / \psi^{\prime}\right)$ for $\psi=\phi_{2} \circ \phi_{1}^{-1}$ coincides in its domain with a solution of the system (14), and its domain is an interval of length at least $\pi$ by Theorem 1.1. Moreover, as the system is autonomous, we can shift $\left(\psi^{\prime}, \psi^{\prime \prime} / \psi^{\prime}\right)$ by $\alpha$, and it will still coincide in its (shifted) 
$14 \mathrm{~J}$. Antezana et al.

domain with a solution $(u(t), v(t))$ of (14). Since $v(0)=0$, it then follows by Lemma 4.3 that for those $k \in \mathbb{Z}$ for which the computation makes sense, we get

$$
\left(\phi_{2} \circ \phi_{1}^{-1}\right)\left(\frac{\pi k}{2}+\alpha\right)-\left(\phi_{2} \circ \phi_{1}^{-1}\right)(\alpha)=\frac{\pi k}{2} .
$$

So, letting $v_{k}=\phi_{1}^{-1}\left(\frac{\pi k}{2}+\alpha\right)$ and $\beta=\phi_{2} \circ \phi_{1}^{-1}(\alpha)$, we get

$$
\phi_{2}\left(V_{k}\right)=\beta-\alpha+\phi_{1}\left(V_{k}\right) .
$$

Therefore, we have that on the points $t \in \mathbb{R} \operatorname{such}$ that $\phi_{1}(t)=\alpha\left(\bmod \frac{\pi}{2}\right)$ it holds that

$$
\phi_{2}(t)=\beta-\alpha+\phi_{1}(t)
$$

Reversing the roles of $\phi_{1}$ and $\phi_{2}$ above, we get that the same holds on the points $t \in \mathbb{R}$ such that $\phi_{2}(t)=\beta\left(\bmod \frac{\pi}{2}\right)$. Here, we need to use the fact that if $\alpha$ is an inflection point of a function $\varphi$, then $\varphi(\alpha)$ is an inflection point of $\varphi^{-1}$.

The proof of Theorem 2.3 is now a consequence of the following modification of a result of de Branges [4, Theorem 24], which is well known to specialists.

Lemma 4.4. Let $E_{1}$ and $E_{2}$ be two Hermite-Biehler functions without real zeros, with phase functions $\phi_{1}$ and $\phi_{2}$, respectively. Suppose that there exist $\alpha, \beta \in \mathbb{R}$ so that

$$
\phi_{2}(t)=\beta-\alpha+\phi_{1}(t)
$$

for all $t \in \mathbb{R}$ such that $\phi_{1}(t)=\alpha\left(\bmod \frac{\pi}{2}\right)$ or $\phi_{2}(t)=\beta\left(\bmod \frac{\pi}{2}\right)$. Then, there exists a nonvanishing real entire function $S$ such that $F \mapsto S F$ is an isometric isomorphism from $H\left(E_{1}\right)$ on to $H\left(E_{2}\right)$.

Proof. Define the Hermite-Biehler functions $\widetilde{E}_{1}$ and $\widetilde{E}_{2}$ by

$$
\widetilde{E}_{1}(z)=\mathrm{e}^{\mathrm{i} \alpha} E_{1}(z) \text { and } \widetilde{E}_{2}(z)=\mathrm{e}^{\mathrm{i} \beta} E_{2}(z) .
$$

If $\widetilde{\phi}_{1}$ and $\widetilde{\phi}_{2}$ denote their corresponding phase functions, then

$$
\widetilde{\phi}_{1}(t)=\widetilde{\phi}_{2}(t),
$$

whenever $t$ is such that $\widetilde{\phi}_{1}(t)=0\left(\bmod \frac{\pi}{2}\right)$ or $\widetilde{\phi}_{2}(t)=0\left(\bmod \frac{\pi}{2}\right)$. Therefore, by Theorem 24 in [4], there exists an entire function $S$, real valued on $\mathbb{R}$, such that

$$
F \mapsto S F,
$$


is an isometric map between the spaces $H\left(\widetilde{E}_{1}\right)$ and $H\left(\widetilde{E}_{2}\right)$. Since $H\left(E_{1}\right)=H\left(\widetilde{E}_{1}\right)$ and $H\left(E_{2}\right)=$ $H\left(\widetilde{E}_{2}\right)$, in the sense of Hilbert spaces, it follows that $S$ induces an isometry between the original spaces $H\left(E_{1}\right)$ and $H\left(E_{2}\right)$. A priori, this function may have real zeros. However, since neither $E_{1}$ nor $E_{2}$ have real zeros, the function $S$ never vanishes. Indeed, the function $S$ satisfies the identity

$$
K_{2}(z, w)=S(z) K_{1}(z, w) \overline{S(w)},
$$

and $K_{j}(x, x)=\frac{1}{\pi} \phi_{j}^{\prime}(x)\left|E_{j}(x)\right|^{2}$ for $j=1,2$ and $x \in \mathbb{R}$. From this it follows that, for every $x \in \mathbb{R}$,

$$
|S(x)|^{2}=\frac{K_{2}(x, x)}{K_{1}(x, x)}=\frac{\phi_{2}^{\prime}(x)\left|E_{2}(x)\right|^{2}}{\phi_{1}^{\prime}(x)\left|E_{1}(x)\right|^{2}} \neq 0 .
$$

Now we can easily deduce Theorem 2.2 from Theorem 2.3.

Proof of Theorem 2.2. Let $F$ be a de Branges GAF defined by the reproducing kernel $K_{1}(z, w)$ of the space $H\left(E_{1}\right)$ and let $G$ be the de Branges GAF defined by the reproducing kernel $K_{2}(z, W)$ of the space $H\left(E_{2}\right)$. It follows from Theorem 2.3 that $S F$ and $G$ have the same covariance kernel and therefore, as they are Gaussian processes, $S F$ and $G$ have the same distribution, but $S$ does not vanish, so we get the result.

\section{Funding}

This work was supported by the Generalitat de Catalunya (project 2014 SGR 289 to J.M.) and the Spanish Ministerio de Economía y Competividad (project MTM2014-51834-P to J.M.)

\section{Acknowledgments}

We thank Joaquim Ortega-Cerdà for fruitful discussions on the subject matter of this article and Àlex Haro for enlightening discussions about the system of ODEs appearing in the last section. We would also like to express our gratitude to the careful referee for all his/her numerous comments and suggestions on how to improve the presentation.

\section{References}

[1] Adler, R. J., and J. E. Taylor. Random Fields and Geometry. Springer Monographs in Mathematics, New York: Springer, 2007.

[2] Ahlfors, L. V. "Cross-ratios and Schwarzian Derivatives in $\mathbf{R}^{\mathbf{n}}$." In Complex Analysis, edited by J. Hersch and A. Huber, 1-15, Basel: Birkhäuser Verlag, 1988. 
16 J. Antezana et al.

[3] Bergman, S. The Kernel Function and Conformal Mapping, revised ed. Mathematical Surveys, No. V. Providence, RI: American Mathematical Society, 1970.

[4] de Branges, L. Hilbert Spaces of Entire Functions. Englewood Cliffs, NJ: Prentice-Hall, Inc., 1968.

[5] Edelman, A., and E. Kostlan. "How many zeros of a random polynomial are real?" Bulletin of the American Mathematical Society (N.S.) 32, no. 1 (1995): 1-37.

[6] Feldheim, N. D. "Zeroes of Gaussian analytic functions with translation-invariant distribution." Israel Journal of Mathematics 195, no. 1 (2013): 317-45.

[7] Hough, J. B., M. Krishnapur, Y.Peres, and B. Virág. Zeros of Gaussian Analytic Functions and Determinantal Point Processes. University Lecture Series, vol. 51. Providence, RI: American Mathematical Society, 2009.

[8] Osgood, B. Old and New on the Schwarzian Derivative. Quasiconformal mappings and analysis (Ann Arbor, MI, 1995), pp. 275-308. New York: Springer, 1998.

[9] Sodin, M. "Zeros of Gaussian analytic functions." Mathematical Research Letters 7, no. 4 (2000): 371-81. 